\title{
ANTE EL CAMBIO DEMOGRÁFICO, ¿NATALIDAD O ROBOTIZACIÓN?
}

La sociedad está siendo testigo de profundos cambios demográficos, tecnológicos y en los estilos de vida que están transformando decisivamente el sistema productivo y las bases mismas de la distribución de la renta. En este contexto, el fuerte descenso de la natalidad refleja en parte, cuando no viene causado por, la incesante deriva de la longevidad. Nada puede reemplazar a los nacimientos en lo que se refiere a aspectos vitales esenciales, pero, como se argumenta en este ensayo, una mejor gestión social e institucional de la edad de cara a la jubilación, así como la adopción de políticas que hagan de la robotización una oportunidad para los trabajadores ayudarían mucho más a mantener los compactos sociales de redistribución de la renta que una natalidad compensatoria de los viejos equilibrios etarios.

\section{Facing demographic change: natality or robotization?}

Society is witnessing deep demographic, technological and lifestyle changes that are decisively transforming productive forces and income distribution schemes. In this context, the steep fall in fertility and birth rates is, in part, a reflection of an ever-increasing longevity, if not its consequence, or, at least, is a sharp contrast with it. Nothing can replace births when it comes to essential aspects of life, both individual and social, but, as I argue in this essay, an improved social and institutional age management before retirement, as well as the adoption of policies that make automation into a true opportunity for workers, would be much more conductive to a better income distribution than pro-natalist policies designed to restore old times age balances.

Palabras clave: longevidad, robots, distribución de la renta.

Keywords: longevity, robots, income distribution.

JEL: J1, O3.

\section{Introducción}

Uno de los fenómenos demográficos que más ha calado en el imaginario social (y político) de las últimas décadas en España es el del colapso de la natalidad.

\footnotetext{
* Director asociado de Afi, Presidente del Consejo de Expertos del Instituto BBVA de Pensiones, Vocal del Foro de Expertos del Instituto Santalucía de Ahorro y Pensiones.

Este artículo se basa en la ponencia del mismo título pronunciada por su autor en las «XXXIII Jornadas de Alicante de Economía Española» el 8 de noviembre de 2018. Agradezco los comentarios del editor de la revista y de un evaluador anónimo que han contribuido a mejorar sensiblemente el borrador original. Todas las limitaciones que subsistan son responsabilidad exclusiva del autor. DOI: https://doi.org/10.32796/ice.2019.908.6834
}

El modelo de la «transición demográfica», proceso cuyo primer inicio se puede identificar en la Europa de entreguerras en el siglo XX (Van de Kaa, 1987), postula que el desarrollo económico y los avances en materia de salud y planificación familiar reducen progresivamente, pero de forma intensa, las tasas de mortalidad y de natalidad hasta estabilizarlas de forma que la población alcance el equilibrio estacionario gobernada por la tasa de reposición generacional que determina una fecundidad de 2,1 hijos por mujer en edad fértil. En una segunda oleada, mediando el enorme cambio de actitudes y valores individuales y sociales desde la finalización de la Segunda Guerra 
Mundial, Van de Kaa constata que en la primera mitad de la década de los ochenta del siglo pasado muchos países europeos habían alcanzado ya tasas de fecundidad inferiores a la de equilibrio generacional.

Estos patrones se están cumpliendo, con excepciones y pautas temporales diferentes y más retardadas en muchas sociedades emergentes y en vías de desarrollo todavía hoy, en todos los países. Pero en Europa se han visto compensados por políticas públicas muy intensas en países como los nórdicos o Francia y, a la vez, reforzados por un auténtico colapso de la natalidad en países como Italia, España y las repúblicas bálticas.

El descenso de la natalidad ha sido durante décadas el resultado lógico de las mejoras en materia de salud pública, avances médicos y, como condición sine qua non, planificación familiar. Todo ello facilitó la incorporación de la mujer al mercado laboral, lo que retroalimentó el proceso. Pero, de ninguna manera, el nivel de reposición generacional es un objetivo que se autorregula. De hecho, especialmente, el proceso de incorporación de la mujer al mercado laboral que muchos países han experimentado, junto a otros factores, está produciendo una «sobrerreacción» de la tasa de fertilidad hacia niveles muy por debajo del nivel de reposición.

Curiosamente, a modo de compensación, viene dándose este aumento incesante de la duración media de la vida, a todas las edades, en lo que se refiere a la "vida restante» (esperanza de vida a una edad cualquiera), y en todos los países. Este alargamiento de la duración de la vida es un fenómeno que viene produciéndose también, con pasmosa regularidad, desde mediados del siglo XIX. En los países evocados, alrededor del año 1900, la esperanza de vida oscilaba todavía alrededor de los 40 años. En la actualidad supera los 80 años. Para muchos países, podría decirse, los niños que no nacen son los años de más que vivimos cada década (Herce, 2018a). Es decir, si totalizamos, para una cohorte cualquiera, las horas de vida adicionales que esta cohorte acumula hasta su desaparición, como consecuencia de una creciente esperanza de vida (a razón de más de 5 horas cada 24), nos encontraríamos con un bonus demográfico equivalente a un mini baby-boom. Con una diferencia muy importante: los recién nacidos no son un recurso productivo humano hasta muchos años después, y mediando una relevante inversión educativa en ellos, mientras que muchas de esas horas añadidas a la vida adulta lo son directamente.

Solo que la percepción social de la creciente longevidad como un proceso compensatorio de la decreciente natalidad, sin que puedan negarse visos de causalidad entre ambos fenómenos (Garrido, 1996), apenas es constatable. En otras palabras, la alarma social que causa la baja natalidad no se corresponde en absoluto con la nula percepción social de la longevidad como una fuente de recursos: los años extra que se añaden a la vida... eso sí, a edades no laborales, a diferencia de lo que sucedía en el siglo XX, cuando las ganancias de vida iban mayoritariamente a edades laborales.

En este contexto, de proporciones e implicaciones enormes, apenas bien percibidas por la sociedad y los decisores políticos que la representan, se despliega ante nuestros ojos la revolución digital. Llevamos décadas conviviendo con los robots. Y no me refiero a las Thermomix. Robots antropomorfos, brazos articulados y robots de software, en el sentido de Turing (1950), que automatizan procesos dotados de inteligencia artificial y actúan en todos los sectores productivos, desde la industria pesada hasta la bioingeniería, desde las ventas al por menor hasta la logística, desde los servicios administrativos hasta los intercambios colaborativos $\mathrm{P} 2 \mathrm{P}$.

La elección entre natalidad y robotización no es tal, ni siquiera un dilema en sí mismo, pero puede convertirse en algo parecido si así lo provocamos, sea por acción o por inacción, deliberada o involuntaria, acierto o error. Pero sí es una invitación a la reflexión y, en su caso, a la acción.

Este artículo es una reflexión abierta basada en referencias recientes y puntuales, no exhaustivas, y en 


\section{GRÁFICO 1}

CURVAS DE SUPERVIVENCIA EN ESPAÑA, 1900-2017

(Ambos sexos, efectivos por edad, generaciones «sintéticas» normalizadas de 100.000 individuos)

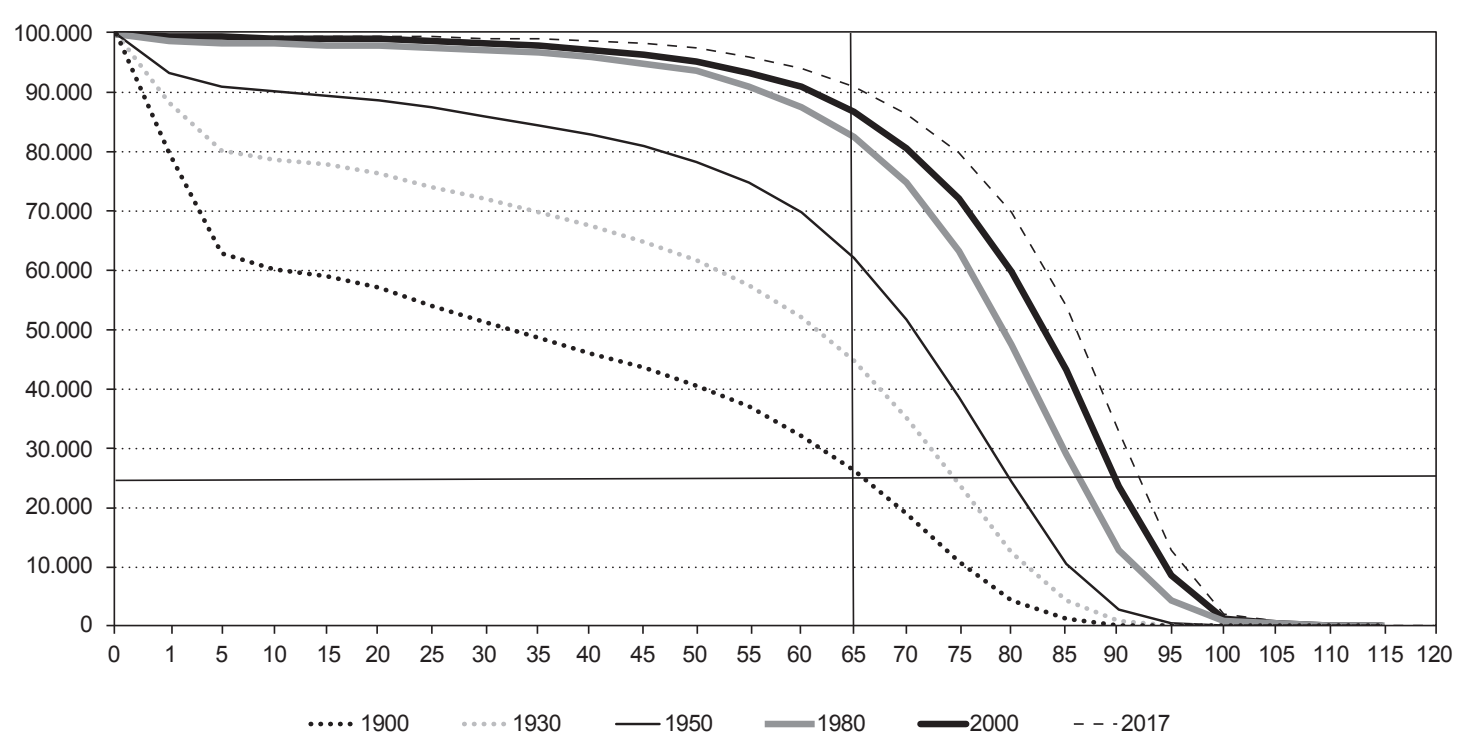

FUENTE: Elaboración propia a partir del INE.

contribuciones personales a debates y conferencias en las que hago frecuente hincapié en que las tendencias de fondo que se describen son potentísimas y no son fruto de errores humanos, sino más bien una mezcla de inmensos aciertos y de comportamientos «racionales», dados los incentivos existentes.

Para abordar la discusión, propongo hablar primero de longevidad, antes que de natalidad, porque solo si se entiende la potencia de lo que está sucediendo en este ámbito, que se enfrenta a una nueva transición hacia vidas extralargas, podrá acomodarse el debate sobre la relación entre natalidad y robotización.

\section{2. ¿Longevidad o envejecimiento?}

En 1900, en España, a los 65 años (edad de jubilación en los escasos sistemas de Seguridad Social entonces existentes en Europa), sobrevivía el 26,2 \% de una generación; en la actualidad sobrevive más del $90 \%$. En aquel mismo año, quienes llegaban a los 65 tenían una esperanza de vida de 9,1 años (unisex), mientras que hoy la esperanza de vida a los 65 años es de más de 20 años.

Esta evidencia pone en cuestión uno de los discursos más prevalentes sobre la edad, tanto en la sociedad como en los medios o entre los responsables políticos: la de que a partir de los 65 años se «es viejo». A esa edad interviene una especie de «reconocimiento» social consistente en descuentos en los autobuses urbanos, en los ferrocarriles, promociones especiales en museos, espectáculos o ese «Eldorado» moderno que han dado en llamar la silver economy.

Dicho de otra manera, si los 65 años son la «tercera edad», ¿qué son los 80 años, o los 100 ? Porque, lo que los datos nos dicen, como se muestra en el Gráfico 1, es que la idea de una "gran edad» a partir de la 


\begin{tabular}{|c|c|c|c|c|}
\hline \multicolumn{5}{|c|}{ CUADRO 1} \\
\hline \multicolumn{5}{|c|}{ EDAD EQUIVALENTE HOY A LOS 65 AÑOS DE 1900* } \\
\hline & \multicolumn{2}{|c|}{$\begin{array}{c}\text { Edad a la que sobrevive el } 26,18 \% \\
\text { de una generación }\end{array}$} & \multicolumn{2}{|c|}{$\begin{array}{c}\text { Edad a la que la esperanza de vida } \\
\text { es de } 9,1 \text { años }\end{array}$} \\
\hline & 1900 & 2017 & 1900 & 2017 \\
\hline 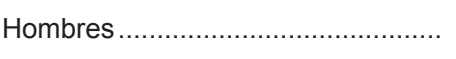 & 65 & 89 & 65 & 79 \\
\hline 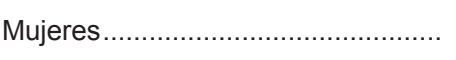 & 65 & 93 & 65 & 82 \\
\hline Ambos sexos & 65 & 91 & 65 & 81 \\
\hline \multicolumn{5}{|c|}{$\begin{array}{l}\text { NOTA: *En } 1900 \text {, a los } 65 \text { años sobrevivía el } 26,18 \% \text { de una generación y la esperanza de vida era de } 9,1 \text { años (unisex). } \\
\text { Edad redondeada al entero más próximo. } \\
\text { FUENTE: Elaboración propia a partir del INE. }\end{array}$} \\
\hline
\end{tabular}

cual al individuo representativo le sucede todo aquello que, a pesar de i) el ambiente prevaleciente, ii) los recursos propios y colectivos disponibles, iii) los estilos de vida generales o iv) su propia genética, lo lleva a la muerte, debe de ser una diana móvil. Nadie sabe cuál es la "gran edad», pero el gráfico aludido es muy elocuente en que si, por casualidad, esa edad, en 1900, fuesen los 65 años, hoy debe estar muy por encima de ella. El lector no tiene más que desplazarse hacia su derecha siguiendo la línea horizontal que corta a la curva de supervivencia de ese año, justamente en la abscisa 65.

Las curvas de supervivencia reflejan la trayectoria de la mortalidad entre 1900 y 2017 y también la enorme ganancia de años de vida que se ha producido en el período. Estas ganancias se han producido en todas las edades y no solo en las edades en las que en el pasado la mortandad infantil era terrible. Hasta el punto de que ya casi no quedan ganancias que realizar a edades laborales (por debajo de los 65 años) y sí mucho por ganar hasta "comprimir» la mortalidad en los 120 años para todos, aunque esto no sucederá de forma tan lineal. Entre otras razones porque el límite de la vida humana ya está siendo puesto en cuestión por muchos investigadores que creen que el envejecimiento puede detenerse e incluso revertirse.

El Cuadro 1 pone números a la dinámica que se desprende del Gráfico 1. Pueden utilizarse dos métricas, al menos, para inferir cuál es hoy la edad equivalente a los 65 años de 1900.

Bajo el criterio del porcentaje que sobrevive a una determinada edad, los 65 años, para encontrar hoy la edad a la que sobrevive el $26,2 \%$ de una generación, lo que en 1900 sucedía a los 65 años, hay que remontarse a los 91 años utilizando las tablas de mortalidad del INE. La otra métrica es la de la esperanza de vida. Es decir, en 1900, a los 65 años, los supervivientes tenían todavía 9,1 años de vida media. Pues bien, para encontrar hoy la edad a la que la esperanza de vida es la misma, hay que remontarse a los 81 años.

La edad equivalente hoy a los 65 años de 1900, es decir, a la que estamos más o menos como entonces a aquella edad, debe estar entre los 81 y los 91 años. Por supuesto, esta es una grosera estimación que necesita muchas puntualizaciones, pero que expresa muy elocuentemente la dinamicidad de lo que deberíamos llamar la «gran edad» en vez de ir añadiendo 
ordinales a los 65 (tercera edad) y, de paso, dejando vacíos de contenido a los precedentes.

Después de este análisis, ¿seguiríamos hablando de «envejecimiento»? O, más bien, ¿empezaríamos a poner el foco en la longevidad como una fuerza dinámica que tira de muchos otros hitos del ciclo vital y que apenas tenemos en cuenta? Con el foco puesto en la longevidad, empezaríamos a entender la causa del denominado «problema de las pensiones», es decir, que no es la falta de nacimientos (lo que puede causar problemas, no menores, de liquidez), sino la incesante extensión de la esperanza de vida sumada a la resistencia a elevar la edad de jubilación (la barrera de los 65 años) la que provoca el problema de solvencia de las pensiones.

\section{3. ¿Niños o robots?}

En el marco recién trazado de una longevidad creciente, ¿dónde se sitúa el clamor por la natalidad?

La primera constatación es que no nacen tantos niños como antes. Según datos oficiales definitivos del INE, en 1975, con una población en España de 35.700.000 habitantes, se produjeron 669.378 nacimientos, esto es, un $18,7 \%$, mientras que, en 2017 , con 46.500 .000 habitantes hubo 393.181 nacimientos, un 8,5\%. En los 42 años transcurridos, pues, la tasa de natalidad ha descendido más de diez puntos. La tasa de fertilidad ha pasado de 2,8 hijos por mujer en edad fértil a 1,3 hijos.

Esto no sucede por casualidad. La evidencia de que la vida es cada vez más larga impregna nuestro ciclo vital de forma material, determinando ajustes en muchas otras decisiones que los individuos y la sociedad adoptan, aunque no en todas (Herce y Del Olmo, 2013). Por ejemplo, se extienden pari passu los períodos educativos, incluidos los obligatorios. Esto valida una de las predicciones básicas de la teoría del capital humano: vidas más largas requieren una mayor acumulación de capital humano. También se retrasan, correspondientemente, las edades de entrada a la actividad laboral, las de maternidad al primer hijo o las de formación del primer hogar. Esto en lo que se refiere a la primera fase del ciclo vital. En la fase madura y final, se constatan retrasos en la edad a la que interviene la incapacidad permanente (a efectos de la percepción de una pensión) o la condición de viudedad (a efectos de percepción de una pensión) por fallecimiento de la pareja. Condiciones estas muy directamente ligadas a los mismos factores que determinan la extensión de la duración de la vida.

Curiosamente, el único indicador que va contracorriente en esta dinámica es el de la edad (efectiva, no legal) media de jubilación. Que, o bien se ha estancado, o incluso ha descendido en las últimas décadas. Esta contradictoria deriva del único factor de ajuste que logra el equilibrio de las fases del ciclo vital en las que se generan recursos y en las que estos se necesitan es inexplicable. Pero ya sabemos que depende de otro tipo de acuerdos que no tienen nada que ver ni con la biología ni con la percepción clara de que la vida dura cada vez más.

En su lugar, preferimos invocar a una natalidad accionable desde la política pública para que nos provea de los efectivos laborales que equilibren los grupos de edad, esa famosa y desmovilizadora pirámide de edad en la que uno solo ve la amenazadora barrera horizontal de los 65 años, ya que no se equilibran los ciclos vitales individuales.

Hay dos tipos de reivindicaciones respecto a la natalidad. La de los responsables políticos que, cual soberanos absolutistas, reivindican «más brazos» para el Estado, de forma que aquellos sostengan a una creciente población jubilada. La segunda reivindicación acerca de la natalidad procede del ámbito mucho más propio a ella que es el de la mujer y, más concretamente, las parejas. Cuando se les interroga acerca de cuántos hijos desearían tener "solventemente», es decir, afrontando los costes y esfuerzos que conlleva su desarrollo y educación, indican sistemáticamente que uno más de los que tienen, desde luego en España (Eurobarometer, 2006).

Esto refleja la necesidad de, ahora sí, instrumentar políticas de conciliación que permitan a las parejas realizar sus aspiraciones reproductivas. Las políticas 
natalistas son viciosas, las de conciliación virtuosas y, en principio, rendirán las últimas un mejor resultado que las primeras. Espero que se aprecie la distinción entre políticas natalistas y políticas de conciliación.

Recapitulemos las dos principales conclusiones de este apartado (apoyados en el apartado previo): i) los niños que no nacen son los años de más que ganamos cada década que pasa, aunque estas ganancias se producen ya a edades no laborales, mientras que los niños, por definición, nacen a edades prelaborales y; ii) las parejas desean tener un hijo más de los que tienen.

La primera expone crudamente una verdad incómoda: no deseamos jubilarnos más tarde convirtiendo estas ganancias de vida en trabajo. Quizá porque no soportamos a nuestros jefes, a nuestros subordinados 0 , simplemente, nuestros trabajos. Estos y aquellos nos desagradan y deseamos terminar cuanto antes nuestra carrera laboral si alguien nos asegura los ingresos sustitutivos del salario, incluso aceptando pérdidas contenidas en las rentas de jubilación. La segunda, más amable, nos puede llevar a la percepción de que los problemas de las pensiones, dada nuestra resistencia a jubilarnos más tarde, se resolverían porque con el tiempo más y más trabajadores entrarían en el circuito de la Seguridad Social, quien sabe si para descubrir más tarde que su jubilación debería afrontar un retraso acrecentado. No parece esta la mejor manera de afrontar los graves problemas de financiación a los que están abocados los sistemas de pensiones en todo el mundo.

¿Y los robots? Porque, si algo está empezando a preocupar a la sociedad, tanto o más que las pensiones, es la perspectiva de que los robots desplacen masivamente a los humanos en la realización de una amplísima gama de tareas y trabajos. El Eurobarómetro es muy elocuente al respecto: «Almost three quarters agree that due to the use of robots and artificial intelligence, more jobs will disappear than new jobs will be created $(74 \%)$, and almost as many agree robots and artificial intelligence steal people's jobs (72 \%)» (Eurobarometer, 2017).
En Jimeno (2019) se puede leer: «By looking at the effects of technological change... taking place at the same time that population ageing, we conclude that it is likely that even though population ageing creates incentives for automation, per capita growth will slow down during the demographic transition that most countries are going through».

No puede decirse que, estando los robots, sobran los niños, claro. Pero si la transición demográfica que se está produciendo es irreversible, el envejecimiento resultante de la resistencia a adaptarse a la "gran edad», un mero efecto estadístico, en realidad, va a obligarnos a adoptar masivamente a los robots. Ello, como muy bien se indica en el párrafo anterior, no va a dejar de tener consecuencias, al menos durante un período transitorio cuya forma, hoy por hoy, no hay manera de discernir. Pero que queda claro, no hay nada fatal en la dicotomía niños-robots, que tampoco es un dilema.

\section{Robots, trabajo y pensiones}

Frey y Osborne (2013) sembraron la alarma. Al menos, en ese humor se interpretó popularmente su contribución pionera a una visión moderna de las consecuencias de la robotización. Desde entonces, la literatura se ha hecho más neutral a medida que se comprueba que, al fin y al cabo, ya llevamos unas cuantas décadas conviviendo con robots. Hasta el punto de que, para algunos, el problema no es que haya demasiados robots, sino que hay pocos. Esto lo reconocen hasta los líderes laboristas (Byrne, 2018).

No es seguro que los robots contribuyan a un aumento neto del empleo, pero está claro que, al menos, ello puede suceder de manera coincidente si se adoptan políticas adecuadas. Todas las grandes disrupciones tecnológicas, hasta el presente, han traído a largo plazo un claro aumento neto del empleo y una expansión de las rentas laborales. Lo que sí es seguro (siempre ha pasado) es que este resultado, de darse, no se dará sin una transición que necesariamente será del tipo «destrucción creativa», à la Schumpeter. 
Ello quiere decir que habrá que instrumentar, con cargo a la productividad extra que se generaría, esquemas que traten de compensar a los desplazados en este proceso - quizá modernizando algunos esquemas actuales orientados a cubrir situaciones asimilables de falta de recursos-. Tampoco deberían despreciarse los mecanismos «de mercado» que puedan ayudar en esta transición. Por ejemplo, el ahorro de los trabajadores, regularmente depositado en forma de activos inmobiliarios o vehículos financieros escasamente vinculados al PIB, podría orientarse hacia activos productivos basados en la digitalización, de forma que una parte de los dividendos de esta fluya naturalmente hacia los trabajadores disminuyendo la necesidad de transferencias.

La disrupción digital lo permea todo. Combinada con el proceso de longevidad extrema al que se aboca la especie, impulsado por la disrupción genética, y los profundos cambios en los estilos de vida, conforma una transformación sin precedentes de la sociedad y las fuerzas productivas.

Todo ello impacta de manera decisiva en los procesos de distribución de la renta. La distribución primaria, para empezar, es decir, el flujo inicial de la renta desde la «boca del manantial» a las arcas de los propietarios de los factores de producción: capital y trabajo. Contra la creencia de que hay una división tajante entre unos y otros, esto no es así, ya que multitud de trabajadores son accionistas de compañías cotizadas y no cotizadas, si bien, no es evidente cómo lograr que los trabajadores acaben participando masivamente de los beneficios de la automatización por la vía «natural» de la propiedad de una parte significativa de estos medios de producción (DeCanio, 2016).

Fuera de los regímenes totalitarios, incluidos los esclavistas y feudales, los trabajadores y accionistas no son clases mutuamente excluyentes, pueden solaparse hasta niveles muy amplios, como sucedería en un mundo caracterizado por un «capitalismo popular». De ahí mi recomendación anterior de que los trabajadores pongan sus ahorros en empresas basadas en robots e inteligencia artificial.
La distribución primaria puede verse muy favorecida a favor de los trabajadores si la revolución digital les sirve de apoyo para apalancar su productividad y, por ende, sus salarios. Pero también puede desproveerlos de una parte relevante de sus recursos primarios, sea por el desplazamiento de los trabajadores del mercado de trabajo o por la depauperación de sus condiciones laborales. En estos casos, el resultado sería la masificación del «trabajo en pobre» (working poors) o el paro masivo.

Sea como fuere, parece ineludible prever una transición en la que los mecanismos de tax-transfer deberán calibrarse con sumo cuidado para ayudar. Ahora bien, habrá que tener cuidado, porque esta transición hacia, espero, un mejor futuro para el empleo puede desembocar también en un cambio, a peor, del paradigma vigente de causación de derechos sociales y económicos (para este párrafo y siguientes: Herce, 2019).

Muy resumidamente. Si como consecuencia de la revolución digital los empleos se pierden o se precarizan de manera significativa, podría emerger la «mera ciudadanía» como base de los derechos económicos y sociales, sustituyendo a la base laboral de los mismos que hoy existe. Esto puede parecer una ventaja y hasta tiene muchos defensores, por ejemplo, los de la renta básica. Pero más bien, en mi opinión al menos, tiene muchos inconvenientes.

Si bien la mera ciudadanía es ya la base de derechos tan importantes como la educación y la sanidad y otros menores, los derechos a pensión, a prestaciones por desempleo, incapacidad transitoria y otros siguen arraigados en una base laboral que liga su cuantía al desempeño laboral de los individuos y cimenta férreamente su participación activa en el esfuerzo productivo y el reparto primario de sus frutos.

La desaparición o erosión severa de la base laboral y su sustitución por la mera ciudadanía como fundamento de los derechos económicos y sociales constituiría un acto de resignación y abandono frente al reto de la robotización y nunca proporcionaría mejores derechos que los actuales. Una sociedad de renta básica 
generalizada sería la realización de un «escenario Piketty-Elysium» en el que una superclase dominaría a una ingente subclase de "trabajadores» alimentada por una renta básica y/o trabajos sociales de baja calidad ${ }^{1}$.

Entre los derechos que más podrían sufrir con este cambio de base de causación (y acumulación) de los mismos se encuentran las pensiones, especialmente las de jubilación.

Por la discusión del apartado 2, ya debería haber quedado claro que el "problema» de las pensiones no lo causan los niños, sino la creciente esperanza de vida bajo la restricción cultural de la barrera de los 65 años. Es importante entender esto, porque, obviamente, la solución en cada escenario perceptivo es muy diferente y un error en la formulación de políticas podría costar muy caro.

En particular, las políticas natalistas por el motivo de que los niños, ya trabajadores, aporten a la financiación de las pensiones, en ausencia de cambios en la tasa de sustitución de aquellas, correrían el riesgo de acabar provocando algún síntoma propio de un esquema piramidal. Esos niños reclamarían más adelante sus pensiones, con todo derecho, y se necesitarían todavía más niños.

Mutatis mutandis, pero no mucho, del mismo tenor y efectos serían las políticas migratorias; en definitiva, políticas para reforzar la fuerza de cotizantes del sistema que, naturalmente, reclamarán derechos futuros de pensión, con las mismas condiciones que aquellos a quienes han financiado. Esta visión del recurso humano, sean niños o inmigrantes, no puede restringirse a un enfoque puramente demográfico, de equilibrio de efectivos en la ratio "cotizantes/pensionistas». Pero, cada vez más, los factores biométricos

\footnotetext{
1 Este escenario es uno de los anticipados por Thomas Piketty (2013), en el que el $1 \%$ de los individuos poseen la riqueza y las fuentes de renta y el $99 \%$ restante apenas una porción de las rentas generadas en la economía cada ejercicio. El término Elysium se refiere al conocido film de ciencia-ficción del mismo nombre en el que se caracteriza a una sociedad de este tipo mediante el recurso a una inmensa subclase social dominada por una elite que lo posee todo (Elysium, 2013).
}

(supervivencia y mortalidad) deben ser tenidos en cuenta en el cálculo de las pensiones y todo capítulo de gasto social vinculado a la edad, no solamente al número de efectivos.

Es indiscutible que tanto los nacimientos como las migraciones son fenómenos demográficos de primera magnitud que responden a motivaciones íntimas de los individuos y sus hogares. Pero, desde el punto de vista social, sin embargo, y menos aún con una lógica utilitarista (niños o inmigrantes para que financien las pensiones, por ejemplo), no pueden ser ni más ni menos deseables de lo que lo son para los individuos directamente concernidos, y solo cuando la realización de estos fenómenos sobrepasara límites considerados críticos por la sociedad (sobrepoblación, tráfico desordenado o inhumano de personas) se justificaría una intervención. Adviértase, no obstante, que ninguna de estas aseveraciones está libre de interpretaciones muy complejas sobre las que es muy difícil establecer consensos.

Existe la creencia de que, por encima de un determinado valor de la ratio cotizantes/pensionistas, las pensiones serán sostenibles, de lo que se derivan las políticas natalistas y el confiado recurso a la inmigración (de ser esta necesaria, se añade). Pero hay una importante matización que hacer: la creciente longevidad, dadas las fórmulas de cálculo de las pensiones, requerirá cada vez más cotizantes por cada pensionista y cada uno de estos nuevos cotizantes (sean niños o inmigrantes) requerirá más nuevos cotizantes cuando se jubile. Como el proceso no se detiene en esta segunda ronda de «repoblación» de las cohortes de soporte, cabe imaginarse cómo el sistema de pensiones se transmuta gradualmente en un esquema cuasi piramidal.

Por último, todo argumento demográfico y/o biométrico sobre las pensiones debe encuadrarse también en el marco del «futuro del trabajo». Ya se ha aludido al problema de la causación de derechos económicos y sociales y la distribución primaria de la renta. Y, de la misma forma que se teme la irrupción masiva de los 


\section{ESQUEMA 1}

TRABAJO NO CONVENCIONAL Y PROTECCIÓN SOCIAL DE LOS TRABAJADORES

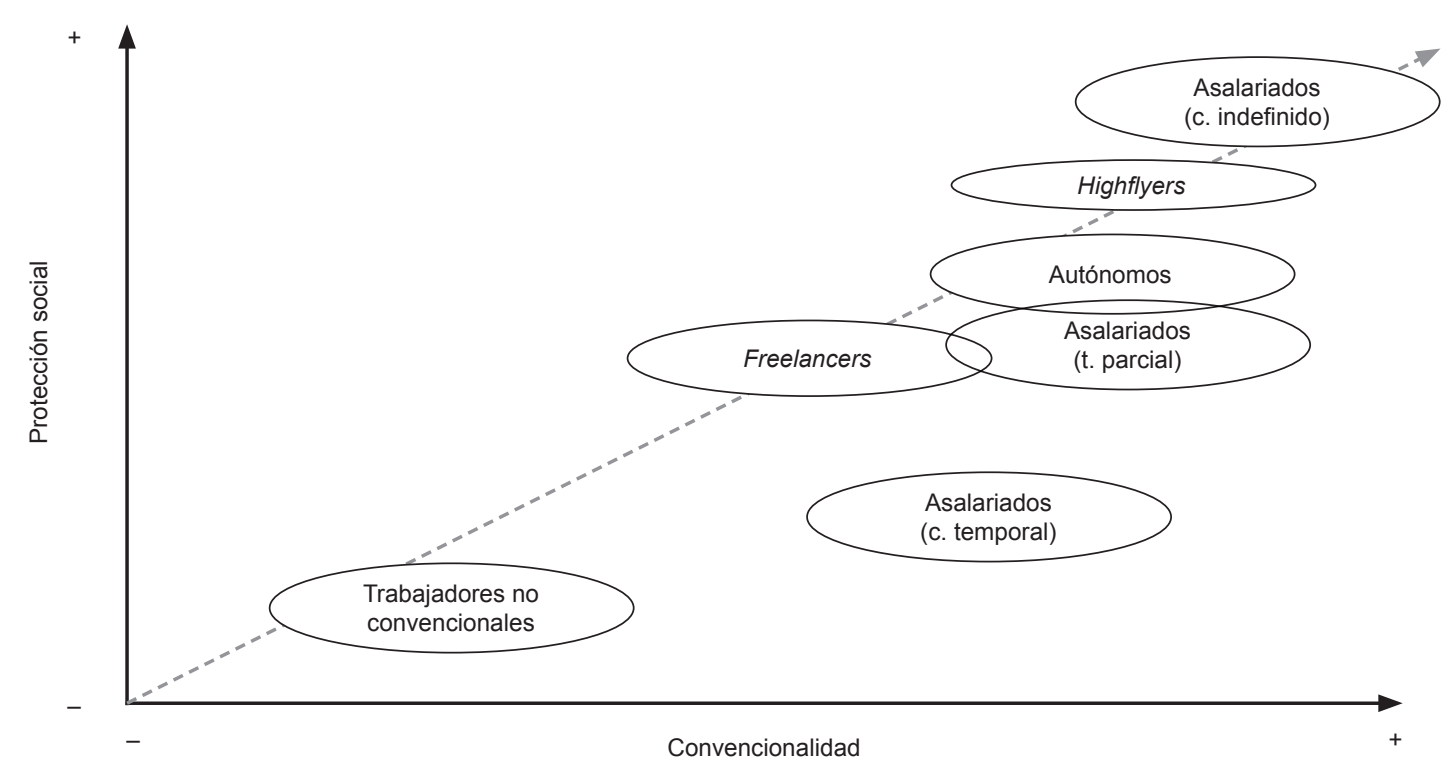

FUENTE: Adaptado de Herce (2018b).

robots en el universo de las tareas desempeñadas por trabajadores humanos con el consiguiente desplazamiento de estos últimos al paro o la precariedad laboral (Frey y Osborne, 2013), cabe pensar que el futuro podría ser mucho más esperanzador en la medida que este desplazamiento de los trabajadores fuese hacia trabajos de más calidad apoyados en robots e inteligencia artificial (Observatorio ADEI, 2017).

Aunque algunos desarrollos actuales, que no invalidan enteramente el anterior desiderátum, relativos a la extensión de la gig economy y el trabajo de plataformas, indican claramente la necesidad de enfocar con cuidado y precisión lo que está sucediendo. En el Esquema 1 se muestra con elocuencia el caso de los trabajos "no convencionales» en lo que se refiere a la protección de sus derechos sociales (Herce, 2018b).

\section{Conclusión}

La sociedad está siendo testigo de profundos cambios demográficos, tecnológicos y en los estilos de vida que están transformando decisivamente el sistema productivo y las bases mismas de la distribución de la renta. Los cambios demográficos se declinan en forma de una fecundidad ultrarreducida y una extensión aparentemente sin límite de la esperanza de vida. La inmigración, que no es un cambio menor, en su aspecto puramente numérico, es una dinámica de suma cero y son otros los aspectos de esta que preocupan o debieran preocupar. Los cambios tecnológicos, en buena medida autónomos respecto a los demográficos, aunque ortogonales a estos, se concentran en una revolución digital plenamente equiparable a la Revolución Neolítica y la Gran Revolución Industrial de los siglos XVIII y XIX, 
más que en la n-sima Revolución Industrial, y prometen trastocar el trabajo, el ocio y los estilos de vida de la población, la gobernanza de la sociedad y, naturalmente las fuerzas productivas y, con ellas, la distribución de la renta.

En este contexto, el fuerte descenso de la natalidad refleja en parte, cuando no viene causado por, la incesante deriva de la longevidad. Nada puede reemplazar a los nacimientos en lo que se refiere a aspectos vitales esenciales, pero, como se argumenta en este ensayo, una mejor gestión social e institucional de la edad de cara a la jubilación lleva ya demasiado tiempo en la agenda social y política. No se trata solo de retrasar la edad de jubilación acompasándola a la evolución de la esperanza de vida. Esta, por otra parte, es la única medida capaz de lograr al mismo tiempo la sostenibilidad y las pensiones. De todas las pensiones, públicas o privadas, de capitalización o de reparto, de prestación o de aportación definida. Pero, además, hay que acabar con una discriminación etaria que se da en muchos ámbitos de la vida social y económica y empezar a ver el tesoro de experiencia y conocimiento, apalancado en la inteligencia artificial, que atesoran los trabajadores maduros.

Las generaciones más jóvenes, si acaso todavía no entienden racionalmente las implicaciones de la demografía y la tecnología (las menos jóvenes menos aún las entienden), registran mejor que las demás algunos cambios, reflejados en sus opciones de formación de hogares y reproductivas, no todas ellas limitadas por la disponibilidad de trabajo, ingresos y tiempo, sino gobernadas por aspiraciones genuinas a configurar su vida de manera diferente empoderadas por la digitalización. Así, sus opciones de actividad productiva, movilidad, posesión de activos o ahorro, no solo no se ven restringidas, sino multiplicadas. Aunque no todos los jóvenes lo han entendido y porfían en las cunetas de los viejos paradigmas laborales y productivos ahondando involuntariamente la trinchera de la precariedad, eso sí, con la inestimable ayuda de los no menos viejos paradigmas educativos y formativos (formación profesional).
Este poderoso cruce de caminos demográficos, tecnológicos y societales se traduce en una clamorosa interpelación a los agentes económicos y sociales y también a los agentes institucionales, de cara a su adaptación a los paradigmas emergentes, a los que deben contribuir a dar forma. Así como induce a la adopción de políticas que hagan de la robotización una oportunidad para los trabajadores. Estas adaptaciones ayudarían mucho más a mantener los compactos sociales de redistribución de la renta que una natalidad compensatoria de los viejos equilibrios etarios.

\section{Referencias bibliográficas}

Byrne, L. (2018). Britain's problem is not too many robots - it's too few. NewStatesman. May 29. Recuperado de https://www.newstatesman.com/politics/business-and-finance/2018/05/britain-s-problem-not-too-many-robots-it-s-toofew

DeCanio, S. J. (2016). Robots and humans - complements or substitutes? Journal of Macroeconomics, 49, 280-291. Recuperado de https://reader.elsevier.com/reader/sd/pii/ S016407041630043X?token=A8304E9C1C963ECE9DF1D6824AC147E6720241C64AB357AFD0E375766B31C6CDDB3D61016886E22CB43744A55180E589

Elysium (2013). Recuperado de https://www.filmaffinity. com/es/film870435.html

Eurobarometer (2006). Childbearing Preferences and Family Issues in Europe. Special Eurobarometer, (253). October. Recuperado de http://ec.europa.eu/commfrontoffice/publicopinion/index.cfm/Survey/getSurveyDetail/instruments/SPECIAL/surveyKy/406/p/5

Eurobarometer (2017). Attitudes towards the impact of digitalisation and automation on daily life. Special Eurobarometer, (460). May. Recuperado de http://ec.europa.eu/commfrontoffice/publicopinion/index.cfm/Survey/getSurveyDetail/instruments/SPECIAL/surveyKy/2160

Frey, C. B. and Osborne, M. A. (2013). The future of employment: How susceptible are jobs to computerisation? Technological Forecasting and Social Change, 2017, 114(C), 254-280. Recuperado de https://www.oxfordmartin.ox.ac.uk/ downloads/academic/The_Future_of_Employment.pdf

Garrido, L. (1996). La revolución reproductiva. En C. Castaño y S. Palacios (Eds.), Salud, dinero y amor. Cómo viven las mujeres españolas de hoy, (pp. 205-238). Madrid: Alianza.

Herce, J. A. (2018a). Longevity and the greyny boom. WD Mi Jubilación. Recuperado de https://www.jubilaciondefuturo. 
es/recursos/doc/pensiones/20160516/en/longevity-and-thegreyny-boom.pdf

Herce, J. A. (2018b). Acceso a la Protección Social de los trabajadores sea cual sea su forma de empleo en el marco del Pilar Europeo de Derechos Sociales. Dictamen para ATA. Recuperado de https://ata.es/wp-content/uploads/2018/05/ Dictamen-ATA-Europa-Completo.pdf

Herce, J. A. (2019). El empleo en el alero y las pensiones al caldero... o no. Economistas, (162-163). Marzo.

Herce, J. A. y Del Olmo, F. (2013). Reinventar la Seguridad Social asegurando la Gran Edad. En J. A. Herce (Dir), Pensiones: Una reforma medular. Fundación de Estudios Financieros y Círculo de Empresarios. Recuperado de https://www. fef.es/publicaciones/papeles-de-la-fundacion/item/273-estudio-pensiones-una-reforma-medular.html
Jimeno, J. F. (2019). Fewer babies and more robots: economic growth in a new era of demographic and technological changes. SERIEs, 1-22. Recuperado de https://link.springer. com/article/10.1007\%2Fs13209-019-0190-z

Observatorio ADEI (2017). El trabajo del futuro. Observatorio ADEI. Recuperado de http://observatorioadei.es/publicaciones/NotaTecnica-El-trabajo-del-futuro.pdf

Piketty, T. (2013). Le Capital au XXIe siècle. París: Seuil. Recuperado de http://piketty.pse.ens.fr/files/Piketty2013IntroChap1.pdf

Turing, A. M. (1950). Computing Machinery and Intelligence. Mind, (49), 433-460.

Van de Kaa, D. J. (1987). Europe's second demographic transition. Population Bulletin, March, 42(1), 1-59. Recuperado de https://estvitalesydemografia.files.wordpress.com/2013/04/ europec2b4s-second-demographic-transition.pdf 


\section{ÚLTIMOS MONOGRÁFICOS PUBLICADOS}

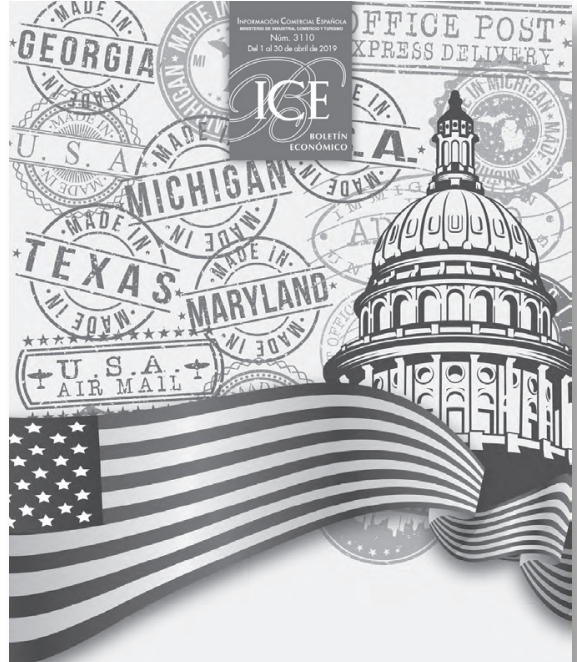

EE UU: de Washington DC a los estados, creando alianzas

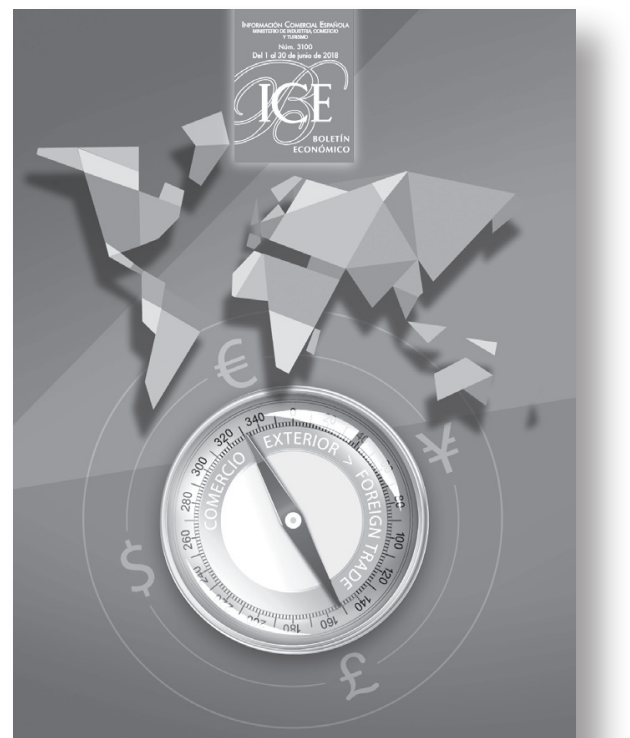

EL SECTOR EXTERIOR EN 2017

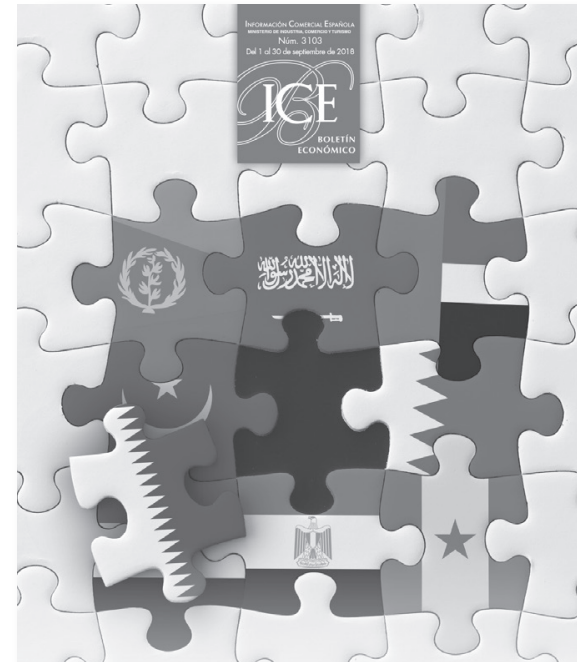

Qatar. Situación y perspectivas ante el bloqueo Evolución del mercado global de divisas

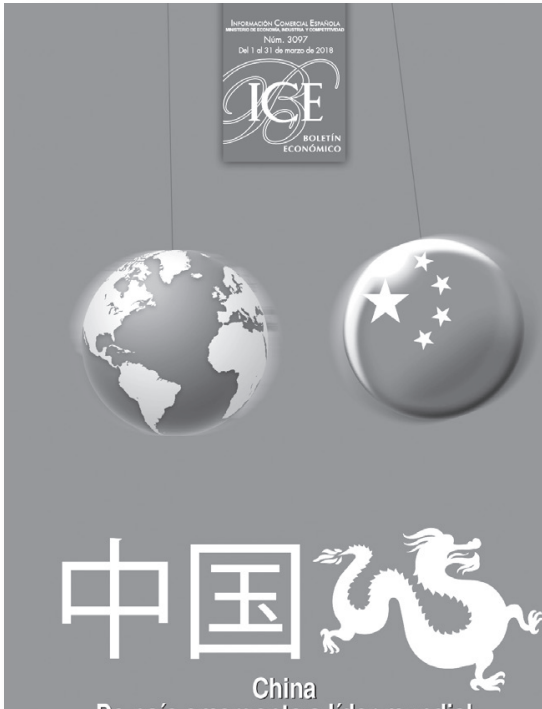

De pais emergente a líder mundifal

Librería virtual y venta www.mincotur.es

Panamá, 1, 28046 Madrid

Telf. 913495129

www.revistasice.com

CentroPublicaciones@mincotur.es 